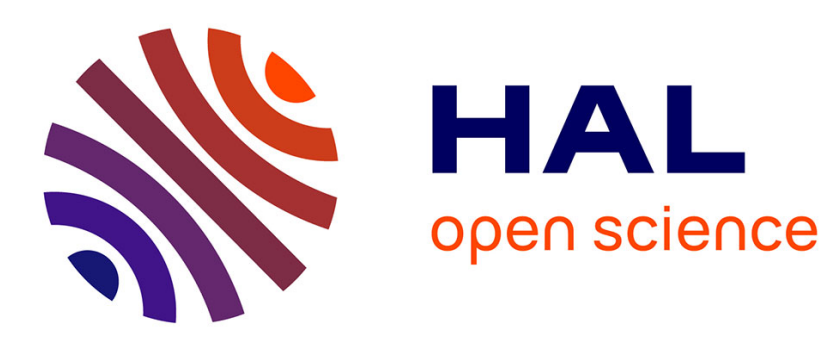

\title{
Unifying Black-Scholes type formulae which involve Brownian last passage times up to a finite horizon
}

D. Madan, Bernard Roynette, Marc Yor

\section{To cite this version:}

D. Madan, Bernard Roynette, Marc Yor. Unifying Black-Scholes type formulae which involve Brownian last passage times up to a finite horizon. 2008. hal-00275490

\author{
HAL Id: hal-00275490 \\ https://hal.science/hal-00275490
}

Preprint submitted on 24 Apr 2008

HAL is a multi-disciplinary open access archive for the deposit and dissemination of scientific research documents, whether they are published or not. The documents may come from teaching and research institutions in France or abroad, or from public or private research centers.
L'archive ouverte pluridisciplinaire HAL, est destinée au dépôt et à la diffusion de documents scientifiques de niveau recherche, publiés ou non, émanant des établissements d'enseignement et de recherche français ou étrangers, des laboratoires publics ou privés. 


\title{
Unifying Black-Scholes type formulae which involve Brownian last passage times up to a finite horizon *
}

\author{
D. Madan \\ Robert H. Smith School of Business, Van Munching Hall, \\ University of Maryland, College Park, MD. 20742, USA \\ email: dbm@rhsmith.umd.edu \\ B. Roynette \\ Université Henri Poincaré, Institut Elie Cartan, BP239, \\ F-54506, Vandoeuvre-les-Nancy Cedex, FRANCE \\ email: bernard.roynette@iecn.u-nancy.fr \\ M. Yor \\ Laboratoire de Probabilités et Modèles Aléatoires \\ Universités Paris VI et VII, 4 Place Jussieu-Case 188 \\ F-75252 Paris Cedex 05 FRANCE \\ email: deaproba@proba.jussieu.fr \\ and \\ Institut Universitaire de France
}

April 19, 2008

\begin{abstract}
The authors recently discovered some interesting relations between the Black-Scholes formula and last passage times of the Brownian exponential martingales, which invites one to seek analogous results for last passage times up to a finite horizon. This is achieved in the present paper, where Yuri's formula, as originally presented in Akahori, Imamura and Yano (2008), is also derived.
\end{abstract}

${ }^{*}$ We are most grateful to J. Akahori for his stimulating suggestion, and more generally to the 3 authors Akahori, Imamura and Yano for providing us with an early version of Yuri's formula. 


\section{Introduction and Notation}

a) Basic Notation. We present some basic notation for the Brownian items we shall deal with throughout the paper, as well as classical results about the laws of first and last passage times for Brownian motion with drift.

For every $\nu \in \mathbb{R}$, we denote the Brownian motion with $\operatorname{drift} \nu$, by $\left(B_{u}^{(\nu)}, u \geq 0\right)$

$$
\left(B_{t}^{(\nu)}, t \geq 0\right):=\left(B_{t}+\nu t, t \geq 0\right)
$$

where $\left(B_{t}, t \geq 0\right)$ is a standard Brownian motion.

b) Filtrations. We shall consider both the usual filtration of the past:

$$
\mathcal{F}_{s}=\sigma\left\{B_{u}, u \leq s\right\}, s \geq 0,
$$

as well as the "past-future", "two parameter filtration"

$$
\mathcal{F}_{s, t}=\sigma\left\{B_{u}, u \leq s ; B_{v}, v \geq t\right\}, 0 \leq s \leq t<\infty .
$$

c) Exponential martingales and the Cameron-Martin formula.

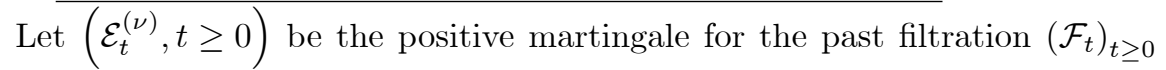
defined by

$$
\left(\mathcal{E}_{t}^{(\nu)}, t \geq 0\right):=\left(\exp \left(\nu B_{t}-\frac{\nu^{2}}{2} t\right), t \geq 0\right)
$$

For $\nu=1$, we shall simply write $\left(\mathcal{E}_{t}\right)$ instead of $\left(\mathcal{E}_{t}^{(1)}\right)$. Throughout the paper, many facts pertaining to $\left(\mathcal{E}_{t}^{(\nu)}\right)$ may be reduced to $\left(\mathcal{E}_{t}\right)$, since by scaling

$$
\left(\mathcal{E}_{t}^{(\nu)}, t \geq 0\right) \stackrel{\text { law }}{=}\left(\mathcal{E}_{\nu^{2} t}, t \geq 0\right)
$$

The Cameron-Martin formula relates the laws of $B^{(\nu)}$ and $B$, as follows:

$$
E\left[F\left(B_{s}^{(\nu)}, s \leq t\right)\right]=E\left[F\left(B_{s}, s \leq t\right) \mathcal{E}_{t}^{(\nu)}\right]
$$

for any positive functional $F$ on $C([0, t] ; \mathbb{R})$.

Let us define for $a \in \mathbb{R}, \nu \in \mathbb{R}$ and $t>0$

$$
\begin{aligned}
T_{a}^{(\nu)}= & \inf \left\{u \geq 0 ; B_{u}^{(\nu)}=a\right\} \\
& (=+\infty \text { if this set is } \emptyset) \\
G_{a}^{(\nu)}= & \sup \left\{u \geq 0 ; B_{u}^{(\nu)}=a\right\} \\
& (=0 \text { if this set is } \emptyset) \\
G_{a}^{(\nu)}(t)= & \sup \left\{u \leq t: B_{u}^{(\nu)}=a\right\} \\
& (=0 \text { if this set is } \emptyset)
\end{aligned}
$$


It is obvious by symmetry that

$$
T_{a}^{(\nu)} \stackrel{(\text { law })}{=} T_{-a}^{(-\nu)} ; G_{a}^{(\nu)}(t) \stackrel{(l a w)}{=} G_{-a}^{(-\nu)}(t)
$$

and that

$$
G_{a}^{(\nu)}(t) \rightarrow_{t \rightarrow \infty} G_{a}^{(\nu)} \text { a.s. }
$$

We recall the classical formulae, for $\nu$ and $a>0$ :

$$
P\left(G_{a}^{(-\nu)}>0\right)=P\left(T_{a}^{(-\nu)}<\infty\right)=\exp (-2 \nu a)
$$

and

$$
\begin{aligned}
P\left(T_{a}^{(\nu)} \in d t\right) & =\frac{a}{\sqrt{2 \pi t^{3}}} \exp \left(-\frac{(a-\nu t)^{2}}{2 t}\right) d t \\
P\left(G_{a}^{(\nu)} \in d t\right) & =\frac{\nu}{\sqrt{2 \pi t}} \exp \left(-\frac{(a-\nu t)^{2}}{2 t}\right) d t
\end{aligned}
$$

whereas, for $a>0$ and $\nu>0$ :

$$
\begin{aligned}
& P\left(T_{a}^{(-\nu)} \in d t\right)=\frac{a}{\sqrt{2 \pi t^{3}}} \exp \left(-\frac{(a+\nu t)^{2}}{2 t}\right) d t \\
& P\left(G_{a}^{(-\nu)} \in d t\right)=\frac{\nu}{\sqrt{2 \pi t}} \exp \left(-\frac{(a+\nu t)^{2}}{2 t}\right) d t
\end{aligned}
$$

In agreement with equation (10) the measures given by formulae (13) and (14) are subprobabilities on $] 0, \infty[$, with total mass : $\exp (-2 \nu a)$. Note that formula (11) may be reduced to its proof for $\nu=0$, thanks to the Cameron-Martin formula (4).

d) A reduced form of the celebrated Black-Scholes formula is the following

$$
E\left[\left(\mathcal{E}_{t}-K\right)^{+}\right]=N\left(-\frac{\log (K)}{\sqrt{t}}+\frac{\sqrt{t}}{2}\right)-K N\left(-\frac{\log (K)}{\sqrt{t}}-\frac{\sqrt{t}}{2}\right)
$$

where $K \geq 0$ and

$$
N(x)=\frac{1}{\sqrt{2 \pi}} \int_{-\infty}^{x} e^{-\frac{y^{2}}{2}} d y
$$

We shall later use the derivative of $N(x)$, denoted by $n(x)$. The formula (15) may be split into two parts

$$
\begin{aligned}
E\left[\mathcal{E}_{t} \mathbf{1}_{\mathcal{E}_{t}>K}\right] & =N\left(-\frac{\log (K)}{\sqrt{t}}+\frac{\sqrt{t}}{2}\right) \\
K P\left(\mathcal{E}_{t}>K\right) & =K N\left(-\frac{\log (K)}{\sqrt{t}}-\frac{\sqrt{t}}{2}\right)
\end{aligned}
$$

A particular case of these equalities in the case $K=1$ is

$$
E\left[\left|\mathcal{E}_{t}-1\right|\right]=2 P\left(B_{1}^{2} \leq \frac{t}{4}\right)
$$


that follows on noting that

$$
E\left[\left(\mathcal{E}_{t}-1\right)^{+}\right]-E\left[\left(\mathcal{E}_{t}-1\right)^{-}\right]=E\left(\mathcal{E}_{t}-1\right)=0
$$

and

$$
E\left[\left(\mathcal{E}_{t}-1\right)^{+}\right]+E\left[\left(\mathcal{E}_{t}-1\right)^{-}\right]=E\left[\left|\mathcal{E}_{t}-1\right|\right]
$$

while

$$
E\left[\left(\mathcal{E}_{t}-1\right)^{ \pm}\right]=P\left(B_{1}^{2} \leq \frac{t}{4}\right) .
$$

e) In Madan, Roynette and Yor (2008b), the following representations of (16),(17) are obtained:

\section{Theorem 0.}

For any $\mathrm{K} \geq 0$ there are the representations

i)

$$
\begin{aligned}
E\left[\left(\mathcal{E}_{t}-K\right)^{+}\right] & =E\left(\mathcal{E}_{t} \mathbf{1}_{\mathcal{E}_{t}>K}\right)-K P\left(\mathcal{E}_{t}>K\right) \\
& =P\left(G_{\log (K)}^{\left(\frac{1}{2}\right)} \leq t\right)
\end{aligned}
$$

ii) For $K \geq 1$

$$
E\left(\mathcal{E}_{t} \mathbf{1}_{\mathcal{E}_{t}>K}\right)+K P\left(\mathcal{E}_{t}>K\right)=P\left(T_{\log (K)}^{\left(\frac{1}{2}\right)} \leq t\right)
$$

while for $K \leq 1$

$$
E\left(\mathcal{E}_{t} \mathbf{1}_{\mathcal{E}_{t}<K}\right)+K P\left(\mathcal{E}_{t}<K\right)=P\left(T_{\log (K)}^{\left(\frac{1}{2}\right)} \leq t\right)
$$

There are at least two possible extensions of Theorem 0.

- One may replace the index $(1 / 2)$ by an arbitrary index $\nu$

- One may replace the last passage times that are for an infinite horizon by the last passage times up to a finite horizon, that is, replace $G_{K}^{(\nu)}$ by $G_{K}^{(\nu)}(t)$.

These extensions shall be demonstrated respectively in Sections 2 and 3.

f) We now explain the genesis of this paper:

(f.1) It was remarked in Madan, Roynette, Yor (2008a,b,c), that for any $\mathbb{R}^{+}$valued continuous local martingale $\left(M_{t}, t \geq 0\right)$ which converges a.s. to 0 , there is the identity

$$
\left(1-\frac{M_{t}}{K}\right)^{+}=P\left(\mathcal{G}_{K} \leq t \mid \mathcal{F}_{t}\right)
$$

where $\mathcal{G}_{K}=\sup \left\{t \geq 0: M_{t}=K\right\}$. Thus the put option value $E\left[\left(K-M_{t}\right)^{+}\right]$ equals $K P\left(\mathcal{G}_{K} \leq t\right)$, which may be considered as an alternative presentation 
of the Black-Scholes formula, e.g. when $M_{t}=\mathcal{E}_{t}=\exp \left(B_{t}-\frac{t}{2}\right)$, (and in this situation, $\left.\mathcal{G}_{K}=G_{\log (K)}^{\left(-\frac{1}{2}\right)} \stackrel{\text { law }}{=} G_{-\log (K)}^{\left(\frac{1}{2}\right)}\right)$, assuming one knows the law of $\mathcal{G}_{K}$ which for $M_{t}=\mathcal{E}_{t}$, is obtained via formula (12). Conversely if one knows

$E\left[\left(K-M_{t}\right)^{+}\right]$for all strikes $K$, and all maturities $t$, then one knows the laws of the $\mathcal{G}_{K}$ 's. See theorem 0 for precise statements in the Brownian framework.

(f.2) It was later suggested to us by J. Akahori (private communication) that, since practically, having access to $\mathcal{G}_{K}$ may be hard, due to the fact that $\mathcal{G}_{K}$ is a "far remote variable", it may be of interest to look for a variant of (23), starting from the computation

$$
P\left(\mathcal{G}_{K}(t) \leq s \mid \mathcal{F}_{s}\right)
$$

for $s<t$, where $\mathcal{G}_{K}(t)=\sup \left\{u \leq t: M_{u}=K\right\}$, and (24) should hopefully be computed explicitly in terms of $M$.

For $M_{t}=\mathcal{E}_{t}$, an answer to this question is given below, in Theorem 3, formula (45), in the improved form of the computation of

$$
P\left(\mathcal{G}_{K}(t) \leq s \mid \mathcal{F}_{s, t}\right)
$$

whereas, in Akahori, Imamura, Yano (2008), an interesting formula (see (47) below) referred to both in Akahori, Imamura, and Yano(2008) and here, as Yuri's formula, expresses $P\left(\mathcal{G}_{K}(t) \leq s\right)$ in a two parameter "Black-Scholes type formula". The main aim of the present paper has been to provide a passage between formulae (45) and (47).

\section{Extension of Theorem 0 to arbitrary index $\nu$}

Theorem 1. (Formulas for an infinite horizon) For all $\nu$ and $K, s>0$

i)

$$
\begin{aligned}
P\left(G_{\log (K)}^{(\nu)}<s\right) & =E\left(\mathcal{E}_{s}^{(2 \nu)} \mathbf{1}_{\mathcal{E}_{s}^{(2 \nu)}>K^{2 \nu}}\right)-K^{2 \nu} P\left(\mathcal{E}_{s}^{(2 \nu)}>K^{2 \nu}\right) \\
& =E\left[\left(\mathcal{E}_{s}^{(2 \nu)}-K^{2 \nu}\right)^{+}\right]
\end{aligned}
$$

We also have that

$$
K^{-2 \nu} P\left(G_{\log (K)}^{(\nu)}<s\right)=E\left[\left(K^{-2 \nu}-\mathcal{E}_{s}^{(-2 \nu)}\right)^{+}\right]
$$

ii) In particular, equation (26) may be improved to

$$
\begin{aligned}
P\left(G_{\log (K)}^{(\nu)}<s \mid \mathcal{F}_{s}\right) & =\left(1-\exp \left(-2 \nu\left(B_{s}^{(\nu)}-\log (K)\right)\right)\right)^{+} \\
& =\left(1-K^{2 \nu} \mathcal{E}_{s}^{(-2 \nu)}\right)^{+} \\
& =K^{2 \nu}\left(K^{(-2 \nu)}-\mathcal{E}_{s}^{(-2 \nu)}\right)^{+}
\end{aligned}
$$


iii) For every $K \geq 0$, if $\nu \log (K)>0$ :

$$
P\left(T_{\log (K)}^{(\nu)} \leq s\right)=E\left(\mathcal{E}_{s}^{(2 \nu)} \mathbf{1}_{\mathcal{E}_{s}^{(2 \nu)}>K^{2 \nu}}\right)+K^{2 \nu} P\left(\mathcal{E}_{s}^{(2 \nu)}>K^{2 \nu}\right)
$$

and if $\nu \log (K)<0$

$$
P\left(T_{\log (K)}^{(\nu)} \leq s\right)=E\left(\mathcal{E}_{s}^{(2 \nu)} \mathbf{1}_{\mathcal{E}_{s}^{(2 \nu)}<K^{2 \nu}}\right)+K^{2 \nu} P\left(\mathcal{E}_{s}^{(2 \nu)}<K^{2 \nu}\right)
$$

Furthermore for $K=1$ and $\nu \neq 0$

$$
\begin{aligned}
P\left(T_{0}^{(\nu)}<s\right) & =E\left(\mathcal{E}_{s}^{(2 \nu)} \mathbf{1}_{\mathcal{E}_{s}^{(2 \nu)}>1}\right)+P\left(\mathcal{E}_{s}^{(2 \nu)}>1\right) \\
& =E\left(\mathcal{E}_{s}^{(2 \nu)} \mathbf{1}_{\mathcal{E}_{s}^{(2 \nu)}<1}\right)+P\left(\mathcal{E}_{s}^{(2 \nu)}<1\right) \\
& =1
\end{aligned}
$$

Proof of theorem 1. For i) and iii) we have by scaling

$$
\frac{1}{4 \nu^{2}} G_{2 a \nu}^{\left(\frac{1}{2}\right)} \stackrel{l a w}{=} G_{a}^{(\nu)}, \frac{1}{4 \nu^{2}} T_{2 a \nu}^{\left(\frac{1}{2}\right)} \stackrel{l a w}{=} T_{a}^{(\nu)}
$$

for all $\nu \neq 0$ and $a$ :

$$
\mathcal{E}_{t}^{(2 \nu)} \stackrel{\text { law }}{=} \mathcal{E}_{t}^{(-2 \nu)} \stackrel{l a w}{=} \mathcal{E}_{4 \nu^{2} t}
$$

and therefore we obtain that

$$
\begin{aligned}
P\left(G_{\log (K)}^{(\nu)}<s\right) & =P\left(\frac{1}{4 \nu^{2}} G_{\log \left(K^{2 \nu}\right)}^{\left(\frac{1}{2}\right)}<s\right)(\text { from }(34)) \\
& =P\left(G_{\log \left(K^{2 \nu}\right)}^{\left(\frac{1}{2}\right)}<4 \nu^{2} s\right) \\
& =E\left[\left(\mathcal{E}_{4 \nu^{2} s}-K^{2 \nu}\right)^{+}\right](\text {from (Theorem 0 (i)) } \\
& =E\left[\left(\mathcal{E}_{s}^{(2 \nu)}-K^{2 \nu}\right)^{+}\right](\text {from (35)) }
\end{aligned}
$$

and this establishes (25). Similar arguments establish (29) and (30). With respect to (33), from (4) we have, from Cameron-Martin

$$
\begin{aligned}
E\left[\mathcal{E}_{s}^{(2 \nu)} \mathbf{1}_{\left.\mathcal{E}_{s}^{(2 \nu)}<1\right]}\right. & =P\left(e^{2 \nu\left(B_{s}+2 \nu s\right)-2 \nu^{2} s}<1\right) \\
& =P\left(e^{-2 \nu B_{s}-2 \nu^{2} s}>1\right) \\
& =P\left(\mathcal{E}_{s}^{(2 \nu)}>1\right)
\end{aligned}
$$

Hence

$$
E\left(\mathcal{E}_{s}^{(2 \nu)} \mathbf{1}_{\mathcal{E}_{s}^{(2 \nu)}>1}\right)=P\left(\mathcal{E}_{s}^{(2 \nu)}<1\right)
$$

Consequently we obtain:

$$
E\left(\mathcal{E}_{s}^{(2 \nu)} \mathbf{1}_{\mathcal{E}_{s}^{(2 \nu)}>1}\right)+P\left(\mathcal{E}_{s}^{(2 \nu)}>1\right)=E\left[\mathcal{E}_{s}^{(2 \nu)} \mathbf{1}_{\mathcal{E}_{s}^{(2 \nu)}<1}\right]+P\left(\mathcal{E}_{s}^{(2 \nu)}<1\right)
$$


and so

$E\left(\mathcal{E}_{s}^{(2 \nu)} \mathbf{1}_{\mathcal{E}_{s}^{(2 \nu)}>1}\right)+P\left(\mathcal{E}_{s}^{(2 \nu)}>1\right)+E\left[\mathcal{E}_{s}^{(2 \nu)} \mathbf{1}_{\mathcal{E}_{s}^{(2 \nu)}<1}\right]+P\left(\mathcal{E}_{s}^{(2 \nu)}<1\right)=1+1=2$

which establishes (31),(32), and (33).

For (26) we note that

$$
\begin{aligned}
E\left[\left(K^{-2 \nu}-\mathcal{E}_{s}^{(-2 \nu)}\right)^{+}\right] & =E\left[\mathcal{E}_{s}^{(-2 \nu)}\left(\frac{K^{-2 \nu}}{\mathcal{E}_{s}^{(-2 \nu)}}-1\right)^{+}\right] \\
& =E\left[\left(K^{-2 \nu} \mathcal{E}_{s}^{(2 \nu)}-1\right)^{+}\right](\text {from }(4)) \\
& =K^{-2 \nu} E\left[\left(\mathcal{E}_{s}^{(2 \nu)}-K^{2 \nu}\right)^{+}\right] \\
& =K^{-2 \nu} P\left(G_{\log (K)}^{(\nu)}<s\right)(\text { from }(25))
\end{aligned}
$$

We shall demonstrate (ii) of theorem 1 in section 3 .

Remark. 1) Taking limits as $s$ tends to 0 and $s$ tends to $\infty$ in (29) and (30) we obtain

- if $\nu \log (K)>0$ :

$$
P\left(G_{\log (K)}^{(\nu)}=0\right)=0=P\left(T_{\log (K)}^{(\nu)}=\infty\right)
$$

- if $\nu \log (K)<0$ :

$$
P\left(G_{\log (K)}^{(\nu)}>0\right)=P\left(T_{\log (K)}^{(\nu)}<\infty\right)=K^{2 \nu}
$$

These results coincide with (10).

Remark 2) The relations (29) and (30) for the case $\nu=0$ (i.e. $\mathcal{E}_{t}^{(\nu)}=$ $\mathcal{E}_{t}^{(0)} \equiv 1$ ) are obtained in the limit as $\nu$ tends to 0 in $(29)$, for example. For $\nu>0$ and $K \geq 1$ we have from (29) that

$$
\begin{aligned}
P\left(T_{\log (K)}^{(\nu)}<s\right)= & E\left(e^{2 \nu\left(B_{s}-\nu s\right)} \mathbf{1}_{\left.e^{2 \nu\left(B_{s}-\nu s\right)}>K^{2 \nu}\right)}+P\left(e^{2 \nu\left(B_{s}-\nu s\right)}>K^{2 \nu}\right)\right. \\
= & E\left(e^{2 \nu\left(B_{s}-\nu s\right)} \mathbf{1}_{B_{s}-\nu s>\log (K)}\right)+P\left(B_{s}-\nu s>\log (K)\right) \\
& \underset{\nu \downarrow 0}{\longrightarrow} 2 P\left(B_{s}>\log (K)\right)
\end{aligned}
$$

On the other hand by (11)

$$
\begin{aligned}
P\left(T_{\log (K)}^{(0)}<s\right) & =\int_{0}^{s} \frac{|\log (K)|}{\sqrt{2 \pi u^{3}}} e^{-\frac{|\log (K)|^{2}}{2 u}} d u \\
& =2 \int_{\frac{|\log (K)|}{\sqrt{s}}}^{\infty} \frac{1}{\sqrt{2 \pi}} e^{-\frac{v^{2}}{2}} d v\left(\text { change variables by } \frac{(\log (K))^{2}}{u}=v^{2}\right) \\
& =2 P\left(B_{s}>\log (K)\right) .
\end{aligned}
$$


A similar analysis holds for (30) as we let $\nu$ tend to 0 .

We now obtain a generalization of (18) for all $\nu$ and $K \geq 0$.

Theorem 2.

For all $K \geq 0$ and all real $\nu$

$E\left[\left(\mathcal{E}_{t}^{(2 \nu)}-K\right)^{ \pm}\right]=(1-K)^{ \pm}+\sqrt{K} E\left(\mathbf{1}_{B_{1}^{2} \leq \nu^{2} t} \exp \left(-\frac{(\log (K))^{2}}{8 B_{1}^{2}}\right)\right)$

Proof. We provide two proofs of theorem 2.

First Proof From relation (3) we need only prove (36) for $\nu=\frac{1}{2}$.

Let $\left(L_{t}^{K}, t \geq 0\right)$ denote the local time at level $K$ for the martingale $\left(\mathcal{E}_{t}, t \geq 0\right)$. From the occupation density formula, we have ${ }^{1}$

$$
\int_{0}^{t} f\left(\mathcal{E}_{s}\right) d\langle\mathcal{E}\rangle_{s}=\int_{0}^{\infty} f(K) L_{t}^{K} d K
$$

for any positive Borel function $f$; since $d\langle\mathcal{E}\rangle_{s}=\left(\mathcal{E}_{s}\right)^{2} d s$, we have that

$$
\int_{0}^{\infty} f(K) E\left(L_{t}^{K}\right) d K=\int_{0}^{t} E\left[f\left(\mathcal{E}_{s}\right)\left(\mathcal{E}_{s}\right)^{2}\right] d s
$$

As (38) holds for all functions $f$ it follows that

$$
E\left(L_{t}^{K}\right)=K \int_{0}^{t} \frac{d s}{\sqrt{2 \pi s}} \exp \left(-\frac{(\log (K)+s)^{2}}{2 s}\right)
$$

where we have used for the density of $\mathcal{E}_{s}$

$$
f_{\mathcal{E}_{s}}(z)=\frac{1}{z} \frac{1}{\sqrt{2 \pi s}} e^{-\frac{1}{2 s}\left(\log (z)+\frac{s}{2}\right)^{2}} \mathbf{1}_{z \geq 0}
$$

On developing the square in the exponential in equation (39) we get

$$
E\left(L_{t}^{K}\right)=\frac{\sqrt{K}}{\sqrt{2 \pi}} \int_{0}^{t} \frac{d s}{\sqrt{s}} \exp \left(-\frac{(\log (K))^{2}}{2 s}-\frac{s}{8}\right)
$$

On the other hand, the Ito-Tanaka formula yields

$$
\begin{aligned}
E\left(\left(\mathcal{E}_{t}-K\right)^{ \pm}\right)= & (1-K)^{ \pm}+\frac{1}{2} E\left(L_{t}^{K}\right) \\
= & (1-K)^{ \pm}+\frac{\sqrt{K}}{2 \sqrt{2 \pi}} \int_{0}^{t} \frac{d s}{\sqrt{s}} \exp \left(-\frac{\log ^{2}(K)}{2 s}-\frac{s}{8}\right) \\
& (\text { from }(41)) \\
= & (1-K)^{ \pm}+ \\
& \frac{\sqrt{K}}{2 \sqrt{2 \pi}} 4 \sqrt{\frac{\pi}{2}} E\left(\mathbf{1}_{4 B_{1}^{2} \leq t} \exp \left(-\frac{\log ^{2}(K)}{8 B_{1}^{2}}\right)\right)
\end{aligned}
$$

\footnotetext{
${ }^{1}$ Changing variables: $K=\exp (x)$ easily yields that $L_{t}^{K}=K L_{t}^{\log (K)}\left(B^{\left(-\frac{1}{2}\right)}\right)$.
} 
where we have employed the density of $B_{1}^{2}$,

$$
f_{B_{1}^{2}}(z)=\frac{1}{\sqrt{2 \pi z}} e^{-\frac{z}{2}} \mathbf{1}_{z \geq 0} .
$$

Formula (36) has thus been proven

\section{Second Proof}

The result (36) is evidently true for $\nu=0$, and as both sides depend only on the absolute value of $\nu$ it suffices to consider just the case $\nu>0$. The derivative with respect to $\nu$, of the right hand side of (36) is

$$
\begin{aligned}
& \frac{\partial}{\partial \nu}\left(\sqrt{K} E\left(\mathbf{1}_{B_{1}^{2} \leq \nu^{2} t} \exp \left(-\frac{(\log (K))^{2}}{8 B_{1}^{2}}\right)\right)\right) \\
= & 2 \sqrt{K} \frac{\partial}{\partial \nu} \int_{0}^{\nu \sqrt{t}} \exp \left(-\frac{(\log (K))^{2}}{8 x^{2}}-\frac{x^{2}}{2}\right) \frac{d x}{\sqrt{2 \pi}} \\
= & \frac{2 \sqrt{K t}}{\sqrt{2 \pi}} \exp \left(-\frac{(\log (K))^{2}}{8 \nu^{2} t}-\frac{\nu^{2} t}{2}\right)
\end{aligned}
$$

On the other hand we may directly evaluate the left hand side of (36) using the Black-Merton-Scholes formula as

$$
E\left[\left(\mathcal{E}_{t}^{(2 \nu)}-K\right)^{+}\right]=N\left(-\frac{\log (K)}{2 \nu \sqrt{t}}+\nu \sqrt{t}\right)-K N\left(-\frac{\log (K)}{2 \nu \sqrt{t}}-\nu \sqrt{t}\right)
$$

Now we take partials with respect to $\nu$ to get

$$
\begin{aligned}
\frac{\partial}{\partial \nu} E\left[\left(\mathcal{E}_{t}^{(2 \nu)}-K\right)^{+}\right]= & n\left(-\frac{\log (K)}{2 \nu \sqrt{t}}+\nu \sqrt{t}\right)\left(\frac{\log (K)}{2 \nu^{2} \sqrt{t}}+\sqrt{t}\right) \\
& -K n\left(-\frac{\log (K)}{2 \nu \sqrt{t}}-\nu \sqrt{t}\right)\left(\frac{\log (K)}{2 \nu^{2} \sqrt{t}}-\sqrt{t}\right)
\end{aligned}
$$

We now recognize that

$$
\begin{aligned}
& n\left(-\frac{\log (K)}{2 \nu \sqrt{t}}+\nu \sqrt{t}\right)=\exp \left(-\frac{(\log (K))^{2}}{8 \nu^{2} t}-\frac{\nu^{2} t}{2}\right) \frac{\sqrt{K}}{\sqrt{2 \pi}} \\
& n\left(-\frac{\log (K)}{2 \nu \sqrt{t}}-\nu \sqrt{t}\right)=\exp \left(-\frac{(\log (K))^{2}}{8 \nu^{2} t}-\frac{\nu^{2} t}{2}\right) \frac{1}{\sqrt{K} \sqrt{2 \pi}}
\end{aligned}
$$

Substituting back into (44) we get that

$$
\frac{\partial}{\partial \nu} E\left[\left(\mathcal{E}_{t}^{(2 \nu)}-K\right)^{+}\right]=\exp \left(-\frac{(\log (K))^{2}}{8 \nu^{2} t}-\frac{\nu^{2} t}{2}\right) \frac{2 \sqrt{K t}}{\sqrt{2 \pi}}
$$

which matches (43), the derivative of the right hand side of (36) with respect to $\nu$. 


\section{Study of Passage times up to a finite horizon}

In the next theorem, we present jointly an expression (45) for the Azéma submartingale $P\left(G_{\log (K)}^{(\nu)}(t)<s \mid \mathcal{F}_{s, t}\right)$, where $t$ may be considered as fixed, and the filtration of reference is $\left(\mathcal{F}_{s, t}\right), s \leq t$, together with Yuri's formula (47)

\section{Theorem 3.}

For every $K \geq 0, \nu \in \mathbb{R}, s \leq t$ :

i)

$P\left(G_{\log (K)}^{(\nu)}(t)<s \mid \mathcal{F}_{s, t}\right)=\left(1-\exp \left[-\frac{2}{t-s}\left(B_{s}^{(\nu)}-\log (K)\right)\left(B_{t}^{(\nu)}-\log (K)\right)\right]\right)^{+}$

ii)

$$
\begin{aligned}
& K^{-2 \nu} P\left(G_{\log (K)}^{(\nu)}(t)<s \mid B_{s}^{(\nu)}=\log (x)\right) \\
= & \mathbf{1}_{x<K}\left[K^{-2 \nu} E\left(\mathcal{E}_{u}^{(-2 \nu)} \mathbf{1}_{B_{u}^{(\nu)}>\log \left(\frac{x}{K}\right)}\right)-x^{-2 \nu} P\left(B_{u}^{(\nu)}<\log \left(\frac{x}{K}\right)\right)\right] \\
& +\mathbf{1}_{x>K}\left[K^{-2 \nu} E\left(\mathcal{E}_{u}^{(-2 \nu)} \mathbf{1}_{B_{u}^{(\nu)}<\log \left(\frac{x}{K}\right)}\right)-x^{-2 \nu} P\left(B_{u}^{(\nu)}>\log \left(\frac{x}{K}\right)\right)\right] \\
(s \leq t & t, u=t-s, x \geq 0)
\end{aligned}
$$

iii) (Yuri's formula)

$$
\begin{aligned}
& P\left(G_{\log (K)}^{(\nu)}(t)<s\right)=E\left[\left(1-K^{2 \nu} \mathcal{E}_{s}^{(2 \nu)}\right)^{+}\right] \\
& +E\left[\operatorname{sgn}\left(1-K^{2 \nu} \mathcal{E}_{s}^{(2 \nu)}\right) \operatorname{sgn}\left(1-K^{2 \nu} \mathcal{E}_{t}^{(2 \nu)}\right)\left(1 \wedge K^{2 \nu} \mathcal{E}_{t}^{(2 \nu)}\right)\right]
\end{aligned}
$$

$\underline{\text { Remark }} 3$ a. Equation (45) is from Bentata and Yor (2008).

b. Yuri's equation (47) is lifted from Akahori, Imamura, and Yano (2008) in the particular case $\nu=-1 / 2$.

c. Theorem 1 , result (ii), is a consequence of equation (45) on letting $t$ tend to infinity and observing that

$$
G_{\log (K)}^{(\nu)}(t) \rightarrow_{t \rightarrow \infty} G_{\log (K)}^{(\nu)}
$$

and

$$
\frac{B_{t}^{(\nu)}}{t} \underset{t \rightarrow \infty}{\rightarrow} \nu \text { a.s }
$$

and then we obtain (27) and (28).

d. An alternative form of equation (46) that we shall use is as follows. For $\nu>0$

$$
\begin{aligned}
& K^{-2 \nu} P\left(G_{\log (K)}^{(\nu)}(t)<s \mid B_{s}^{(\nu)}=\log (x)\right) \\
= & \left(K^{-2 \nu}-x^{-2 \nu}\right)^{+}+ \\
& \left(\mathbf{1}_{x<K}-\mathbf{1}_{x>K}\right) \times \\
& {\left[K^{-2 \nu} E\left(\mathcal{E}_{u}^{(-2 \nu)} \mathbf{1}_{B_{u}^{(\nu)}>\log \left(\frac{x}{K}\right)}\right)-x^{-2 \nu} P\left(B_{u}^{(\nu)}<\log \left(\frac{x}{K}\right)\right)\right] }
\end{aligned}
$$


while for $\nu<0$

$$
\begin{aligned}
& K^{-2 \nu} P\left(G_{\log (K)}^{(\nu)}(t)<s \mid B_{s}^{(\nu)}=\log (x)\right) \\
= & \left(K^{-2 \nu}-x^{-2 \nu}\right)^{+}+ \\
& \left(\mathbf{1}_{x<K}-\mathbf{1}_{x>K}\right) \times \\
& {\left[x^{-2 \nu} P\left(B_{u}^{(\nu)}>\log \left(\frac{x}{K}\right)\right)-K^{-2 \nu} E\left(\mathcal{E}_{u}^{(-2 \nu)} \mathbf{1}_{B_{u}^{(\nu)}<\log \left(\frac{x}{K}\right)}\right)\right] }
\end{aligned}
$$

One goes from (46) to equations (48), (49) on observing that

$$
P\left(B_{u}^{(\nu)}>\log \left(\frac{x}{K}\right)\right)=1-P\left(B_{u}^{(\nu)}<\log \left(\frac{x}{K}\right)\right)
$$

and

$$
E\left(\mathcal{E}_{u}^{(-2 \nu)} \mathbf{1}_{B_{u}^{(\nu)}<\log \left(\frac{x}{K}\right)}\right)=1-E\left(\mathcal{E}_{u}^{(-2 \nu)} \mathbf{1}_{B_{u}^{(\nu)}>\log \left(\frac{x}{K}\right)}\right) .
$$

Proof of theorem 3.

a. Proof of equation (45) (See also Bentata and Yor (2008)) We have from the definition of $G_{l}^{(\nu)}(t)$, for $l \in \mathbb{R}$ :

$$
\left(G_{l}^{(\nu)}(t)<s\right)=A_{s, t}^{+} \cup A_{s, t}^{-}
$$

where

$$
\begin{aligned}
& A_{s, t}^{+}=\{\forall u \in] s, t\left[, B_{u}^{(\nu)}>l\right\} \\
& A_{s, t}^{-}=\{\forall u \in] s, t\left[, B_{u}^{(\nu)}<l\right\}
\end{aligned}
$$

Therefore

$$
\begin{aligned}
& P\left(G_{l}^{(\nu)}(t)<s \mid \mathcal{F}_{s, t}\right)=P\left(A_{s, t}^{+} \mid \mathcal{F}_{s, t}\right)+P\left(A_{s, t}^{-} \mid \mathcal{F}_{s, t}\right) \\
= & \mathbf{1}_{B_{s}^{(\nu)}>l} P\left(\inf _{s \leq u \leq t}\left(B_{u}^{(\nu)}-B_{s}^{(\nu)}\right)>l-B_{s}^{(\nu)} \mid B_{t}^{(\nu)}-B_{s}^{(\nu)}\right) \\
& +\mathbf{1}_{B_{s}^{(\nu)}<l} P\left(\sup _{s \leq u \leq t}\left(B_{u}^{(\nu)}-B_{s}^{(\nu)}\right)<l-B_{s}^{(\nu)} \mid B_{t}^{(\nu)}-B_{s}^{(\nu)}\right)
\end{aligned}
$$

Thus we need to compute for $l$, and $\lambda=l-B_{s}^{(\nu)}$ whereby, for $\lambda(\lambda-m)>0$

$$
\begin{aligned}
& \mathbf{1}_{B_{s}^{(\nu)}>l} P\left(\inf _{s \leq u \leq t}\left(B_{u}^{(\nu)}-B_{s}^{(\nu)}\right)>l-B_{s}^{(\nu)} \mid B_{t}^{(\nu)}-B_{s}^{(\nu)}=m\right) \\
= & 1_{B_{s}^{(\nu)}>l} P\left(T_{\lambda}^{(0)}>t-s \mid B_{t-s}=m\right)
\end{aligned}
$$

and

$$
\begin{aligned}
& \mathbf{1}_{B_{s}^{(\nu)}<l} P\left(\sup _{s \leq u \leq t}\left(B_{u}^{(\nu)}-B_{s}^{(\nu)}\right)<l-B_{s}^{(\nu)} \mid B_{t}^{(\nu)}-B_{s}^{(\nu)}=m\right) \\
= & \mathbf{1}_{B_{s}^{(\nu)}<l} P\left(T_{\lambda}^{(0)}>t-s \mid B_{t-s}=m\right)
\end{aligned}
$$


We note that the quantities in equations (52) and (53) depend only on $t-s$ and not the pair $(s, t)$. Furthermore, the law of the bridge from $a$ to $b$ over the time $[0, u]$ of a Brownian motion with drift $\nu$ is independent of $\nu$. We see that we need to compute

$$
P\left(T_{\lambda}^{(0)}>t-s \mid B_{t-s}=m\right)=1-P\left(T_{\lambda}^{(0)}<t-s \mid B_{t-s}=m\right)
$$

and it seems to be a well known fact that:

$$
P_{0 \rightarrow m}^{(u)}\left(T_{\lambda}<u\right)=\exp \left(-\frac{2(\lambda(\lambda-m))^{+}}{u}\right)
$$

where $P_{0 \rightarrow m}^{(u)}$ denotes the law of a Brownian bridge of length $u$ starting at 0 and ending at $m$. Putting together (51),(52),(53) and (54) with $\lambda=l-B_{s}^{(\nu)}$ and $m=B_{t}^{(\nu)}-B_{s}^{(\nu)}$ we obtain (45)

We comment upon (54): it is a simple application of the symmetry principle whereby

$$
\begin{aligned}
P_{0 \rightarrow m}^{(u)}\left(T_{\lambda}>u\right) & =1-P_{0 \rightarrow m}^{(u)}\left(T_{\lambda}<u\right) \\
& =\frac{\frac{1}{\sqrt{2 \pi u}} \exp \left(-\frac{m^{2}}{2 u}\right)-\frac{1}{\sqrt{2 \pi u}} \exp \left(-\frac{(2 \lambda-m)^{2}}{2 u}\right)}{\frac{1}{\sqrt{2 \pi u}} \exp \left(-\frac{m^{2}}{2 u}\right)} \\
& =1-\exp \left(-\frac{(2 \lambda-m)^{2}}{2 u}+\frac{m^{2}}{2 u}\right) \\
& =1-\exp \left(-\frac{2(\lambda(\lambda-m))^{+}}{u}\right) .
\end{aligned}
$$

In fact, since formula (54) plays a key role in our proof of formula (45), we feel it is relevant to give some references where it also appears: Guasoni (2004), formulae (10)-(11), p.85, Pagès ((2008), Proposition 7.3) in his discussion of the Brownian bridge method for simulating the continuous Euler scheme, Pitman (1999) while deriving the law of the local time at 0 for a Brownian bridge, Yor (1997) while discussing Seshadri's (1988) identities, page 11.

b. Proof of equation (46). We begin by writing

$$
B_{t}^{(\nu)} \stackrel{\operatorname{law}}{=} \widetilde{B}_{s}^{(\nu)}+B_{t-s}^{(\nu)}=\log (x)+B_{u}^{(\nu)} \text { with } u=t-s .
$$


We may then write with $C=\log \left(\frac{x}{K}\right)$

$$
\begin{aligned}
P\left(G_{\log (K)}^{(\nu)}(t)<s \mid \mathcal{F}_{s}\right)= & E\left[1-\exp \left(\frac{2}{u}\left(\log \frac{x}{K}\right)\left(\log \frac{x}{K}+B_{u}^{(\nu)}\right)\right)\right] \operatorname{from}(45) \\
= & \mathbf{1}_{x<K} P\left(B_{u}^{(\nu)}<\log \frac{K}{x}\right)+\mathbf{1}_{x>K} P\left(B_{u}^{(\nu)}>\log \frac{K}{x}\right) \\
& -\mathbf{1}_{x<K} E\left(\mathbf{1}_{B_{u}^{(\nu)}<\log \frac{K}{x}} \exp \left(-\frac{2 C}{u}\left(C+B_{u}^{(\nu)}\right)\right)\right) \\
& -\mathbf{1}_{x>K} E\left(\mathbf{1}_{B_{u}^{(\nu)}>\log \frac{K}{x}} \exp \left(-\frac{2 C}{u}\left(C+B_{u}^{(\nu)}\right)\right)\right) \\
& :=\alpha+\beta-\gamma-\delta
\end{aligned}
$$

We now make the computations for $\alpha, \beta, \gamma$ and $\delta$.

$$
\begin{aligned}
\alpha & =\mathbf{1}_{x<K} P\left(B_{u}^{(\nu)}<\log \left(\frac{K}{x}\right)\right) \\
& =\mathbf{1}_{x<K} E\left(\mathbf{1}_{B_{u}^{(\nu)}<\log \frac{K}{x}} \cdot \frac{\mathcal{E}_{u}^{(-2 \nu)}}{\mathcal{E}_{u}^{(-2 \nu)}}\right) \\
& =\mathbf{1}_{x<K} E\left(\mathbf{1}_{B_{u}-\nu u<\log \left(\frac{K}{x}\right)} e^{2 \nu B_{u}-2 \nu^{2} u}\right) \quad(\text { from (4)) } \\
& =\mathbf{1}_{x<K} E\left(\mathbf{1}_{-B_{u}-\nu u<\log \left(\frac{K}{x}\right)} e^{-2 \nu B_{u}-2 \nu^{2} u}\right) \quad\left(\text { since } B_{u} \stackrel{\text { law }}{=}-B_{u}\right) \\
& =\mathbf{1}_{x<K} E\left(\mathbf{1}_{B_{u}+\nu u>\log \frac{x}{K}} \mathcal{E}_{u}^{(-2 \nu)}\right) \\
& =\mathbf{1}_{x<K} E\left(\mathbf{1}_{B_{u}^{(\nu)}>\log \frac{x}{K}} \mathcal{E}_{u}^{(-2 \nu)}\right)
\end{aligned}
$$

Similarly one shows that

$$
\begin{aligned}
\beta & =\mathbf{1}_{x>K} P\left(B_{u}^{(\nu)}>\log \left(\frac{K}{x}\right)\right) \\
& =\mathbf{1}_{x>K} E\left(\mathbf{1}_{B_{u}^{(\nu)}<\log \left(\frac{x}{K}\right)} \mathcal{E}_{u}^{(-2 \nu)}\right)
\end{aligned}
$$


For $\gamma$ we have that

$$
\begin{aligned}
\gamma= & \mathbf{1}_{x<K} E\left(\mathbf{1}_{B_{u}^{(\nu)}<\log \left(\frac{K}{x}\right)} \exp \left(-\frac{2 C}{u}\left(C+B_{u}^{(\nu)}\right)\right)\right) \\
= & \mathbf{1}_{x<K} E\left(\mathbf{1}_{B_{u}^{(\nu)}<\log \left(\frac{K}{x}\right)} \exp \left(-\frac{2 C}{u}\left(C+B_{u}^{(\nu)}\right)\right) \frac{\mathcal{E}_{u}^{(-2 \nu)}}{\mathcal{E}_{u}^{(-2 \nu)}}\right) \\
= & \mathbf{1}_{x<K} E\left(\mathbf{1}_{B_{u}^{(-\nu)}<\log \left(\frac{K}{x}\right)} \exp \left(-\frac{2 C}{u}\left(C+B_{u}^{(-\nu)}\right)+2 \nu B_{u}-2 \nu^{2} u\right)\right) \text { from (4)) } \\
= & \mathbf{1}_{x<K} \exp \left(-\frac{2 C^{2}}{u}+2 C \nu-2 \nu^{2} u\right) E\left(\mathbf{1}_{B_{u}<-C+\nu u} \exp \left(-\frac{B_{u}}{u}(2 C-2 \nu u)\right)\right) \\
= & \mathbf{1}_{x<K} \exp \left(-\frac{2 C^{2}}{u}+2 C \nu-2 \nu^{2} u\right) \int_{-\infty}^{-C+\nu u} \frac{d y}{\sqrt{2 \pi u}} e^{-\frac{y^{2}}{2 u}-\frac{y}{u}(2 C-2 \nu u)} \\
= & \mathbf{1}_{x<K} \exp \left(-\frac{2 C^{2}}{u}+2 C \nu-2 \nu^{2} u+\frac{1}{2 u}(2 C-2 \nu u)^{2}\right) \int_{-\infty}^{-C+\nu u} \frac{d y}{\sqrt{2 \pi u}} e^{-\frac{1}{2 u}(y+2 C-2 \nu u)^{2}} \\
= & \mathbf{1}_{x<K} \exp (-2 C \nu) \int_{-\infty}^{C-\nu u} \frac{d z}{\sqrt{2 \pi u}} e^{-\frac{z^{2}}{2}} \\
& (\text { after the change of variable } y+2 C-2 \nu u=z) \\
= & \mathbf{1}_{x<K} K^{2 \nu} x^{-2 \nu} P\left(B_{u}^{(\nu)}<C\right)
\end{aligned}
$$

A similar computation yields that

$$
\begin{aligned}
\delta & =\mathbf{1}_{x>K} E\left(\mathbf{1}_{B_{u}^{(\nu)}>\log \left(\frac{K}{x}\right)} \exp \left(-\frac{2 C}{u}\left(C+B_{u}^{(\nu)}\right)\right)\right) \\
& =\mathbf{1}_{x>K} K^{2 \nu} x^{-2 \nu} P\left(B_{u}^{(\nu)}>C\right)
\end{aligned}
$$

Employing the results (56) to (59) back in equation (55) yields (ii) of Theorem 3 .

c. Deriving Yuri's formula (47)

We work with the case $\nu<0$. The result for $\nu>0$ may be deduced on utilizing

$$
G_{\log (K)}^{(\nu)}(t) \stackrel{\operatorname{law}}{=} G_{-\log (K)}^{(-\nu)}(t) .
$$

We shall begin with the equivalent form (49). In the following $\log (x)$ denotes the random variable $\widetilde{B}_{s}+\nu s$ and $x^{-2 \nu}$ denotes the random variable $\mathcal{E}_{s}^{(-2 \nu)}=$ $\exp \left(-2 \nu \widetilde{B}_{s}-2 \nu^{2} s\right)$. Furthermore the random variable $B_{u}$, for $u=t-s$, is 
independent of these random variables. We are given that

$$
\begin{aligned}
& K^{-2 \nu} P\left(G_{\log (K)}^{(\nu)}(t)<s\right)=\widetilde{E}\left(\left(K^{-2 \nu}-x^{-2 \nu}\right)^{+}\right) \\
& -K^{-2 \nu} \widetilde{E}\left(\mathbf{1}_{x<K} E\left(\mathcal{E}_{u}^{(-2 \nu)} \mathbf{1}_{B_{u}^{(\nu)}<\log \left(\frac{x}{K}\right)}\right)\right) \\
& +\widetilde{E}\left(\mathbf{1}_{x<K} x^{-2 \nu} P\left(B_{u}^{(\nu)}>\log \left(\frac{x}{K}\right)\right)\right) \\
& +K^{-2 \nu} \widetilde{E}\left(\mathbf { 1 } _ { x > K } E \left(\mathcal{E}_{u}^{(-2 \nu)} \mathbf{1}_{\left.\left.B_{u}^{(\nu)}<\log \left(\frac{x}{K}\right)\right)\right)}\right.\right. \\
& -\widetilde{E}\left(\mathbf{1}_{x>K} x^{-2 \nu} P\left(B_{u}^{(\nu)}>\log \left(\frac{x}{K}\right)\right)\right) \\
: & =a-b+c+d-e
\end{aligned}
$$

On the other hand, Yuri's formula asserts that

$$
\begin{aligned}
& K^{-2 \nu} P\left(G_{\log (K)}^{(\nu)}(t)<s\right)=E\left(\left(K^{-2 \nu}-\mathcal{E}_{s}^{(-2 \nu)}\right)^{+}\right) \\
& -K^{-2 \nu} P\left(\mathcal{E}_{s}^{(-2 \nu)}<K^{-2 \nu}, \mathcal{E}_{t}^{(-2 \nu)}>K^{-2 \nu}\right) \\
& +E\left(\mathbf{1}_{\mathcal{E}_{s}^{(-2 \nu)}<K^{-2 \nu}} \mathbf{1}_{\mathcal{E}_{t}^{(-2 \nu)}<K^{-2 \nu}} \mathcal{E}_{t}^{(-2 \nu)}\right) \\
& +K^{-2 \nu} P\left(\mathcal{E}_{s}^{(-2 \nu)}>K^{-2 \nu}, \mathcal{E}_{t}^{(-2 \nu)}>K^{-2 \nu}\right) \\
& -E\left(\mathbf{1}_{\mathcal{E}_{s}^{(-2 \nu)}>K^{-2 \nu}} \mathbf{1}_{\mathcal{E}_{t}^{(-2 \nu)}<K^{-2 \nu}} \mathcal{E}_{t}^{(-2 \nu)}\right) \\
& :=a^{\prime}-b^{\prime}+c^{\prime}+d^{\prime}-e^{\prime}
\end{aligned}
$$

The equality of $a$ and $a^{\prime}$ is clear. We now examine the other terms.

- Analysis of the term $b$.

$$
\begin{aligned}
b= & K^{-2 \nu} \widetilde{E}\left(\mathbf{1}_{x<K} E\left(\mathcal{E}_{u}^{(-2 \nu)} \mathbf{1}_{B_{u}^{(\nu)}<\log \left(\frac{x}{K}\right)}\right)\right) \\
= & K^{-2 \nu} \widetilde{E}\left(\mathbf{1}_{\widetilde{\mathcal{E}}_{s}^{(-2 \nu)}<K^{-2 \nu}} E\left(\mathcal{E}_{u}^{(-2 \nu)} \mathbf{1}_{\exp \left(-2 \nu B_{u}-2 \nu^{2} u\right)<K^{2 \nu} \exp \left(-2 \nu \widetilde{B}_{s}-2 \nu^{2} s\right)}\right)\right) \\
= & K^{-2 \nu} \widetilde{E}\left(\mathbf{1}_{\widetilde{\mathcal{E}}_{s}^{(-2 \nu)}<K^{-2 \nu}} P\left(\exp \left(-2 \nu B_{u}+2 \nu^{2} u\right)<K^{2 \nu} \exp \left(-2 \nu \widetilde{B}_{s}-2 \nu^{2} s\right)\right)\right) \text { by }(4) \\
= & K^{-2 \nu} \widetilde{E}\left(\mathbf{1}_{\widetilde{\mathcal{E}}_{s}^{(-2 \nu)}<K^{-2 \nu}} P\left(\exp \left(2 \nu\left(B_{u}-\widetilde{B}_{s}\right)-2 \nu^{2} t\right)>K^{-2 \nu}\right)\right. \\
= & K^{-2 \nu} P\left(\mathcal{E}_{s}^{(-2 \nu)}<K^{-2 \nu}, \mathcal{E}_{t}^{(-2 \nu)}>K^{-2 \nu}\right) \\
& \left(\text { since } B_{u} \stackrel{l a w}{=} B_{-u} \text { and replacing } \widetilde{B}_{s} \text { by } B_{s}\right) \\
= & b^{\prime}
\end{aligned}
$$

- The equality of $d$ and $d^{\prime}$ follows a similar analysis. 
- Analysis of the term c.

$$
\begin{aligned}
c & =\widetilde{E}\left(\mathbf{1}_{x<K} x^{-2 \nu} P\left(B_{u}^{(\nu)}>\log \left(\frac{x}{K}\right)\right)\right) \\
& =\widetilde{E}\left(\mathbf{1}_{\widetilde{\mathcal{E}}_{s}^{(-2 \nu)}<K^{-2 \nu}} \widetilde{\mathcal{E}}_{s}^{(-2 \nu)} E\left[\frac{\mathcal{E}_{u}^{(-2 \nu)}}{\mathcal{E}_{u}^{(-2 \nu)}} \mathbf{1}_{\mathcal{E}_{u}^{(-2 \nu)}>K^{-2 \nu}} \widetilde{\mathcal{E}}_{s}^{(-2 \nu)}\right]\right) \\
= & \widetilde{E}\left(\mathbf{1}_{\widetilde{\mathcal{E}}_{s}^{(-2 \nu)}<K^{-2 \nu}} \widetilde{\mathcal{E}}_{s}^{(-2 \nu)} \exp \left(2 \nu B_{u}-2 \nu^{2} u\right) \mathbf{1}_{\left.\exp \left(-2 \nu B_{u}+2 \nu^{2} u\right)>K^{-2 \nu} \widetilde{\mathcal{E}}_{s}^{(-2 \nu)}\right)}\right. \\
= & \widetilde{E}\left(\mathbf{1}_{\widetilde{\mathcal{E}}_{s}^{(-2 \nu)}<K^{-2 \nu}} \exp \left(-2 \nu\left(\widetilde{B}_{s}-B_{u}\right)-2 \nu^{2} t\right) \mathbf{1}_{\exp \left(-2 \nu\left(\widetilde{B}_{s}-B_{u}\right)-2 \nu^{2} t\right) \leq K^{-2 \nu}}\right) \\
= & E\left(\mathbf{1}_{\mathcal{E}_{s}^{(-2 \nu)}<K^{-2 \nu}} \exp \left(-2 \nu B_{t}-2 \nu^{2} t\right) \mathbf{1}_{\mathcal{E}_{t}^{(-2 \nu)}<K^{-2 \nu}}\right)(\text { from }(4)) \\
& \text { and replacing } \widetilde{B}_{s} \text { by } B_{s} \text { and } B_{u} \text { by }\left(-B_{u}\right) \\
= & E\left(\mathbf{1}_{\mathcal{E}_{s}^{(-2 \nu)}<K^{-2 \nu}} \mathbf{1}_{\mathcal{E}_{t}^{(-2 \nu)}<K^{-2 \nu}} \mathcal{E}_{t}^{(-2 \nu)}\right) \\
= & c^{\prime}
\end{aligned}
$$

The equality of $e$ and $e^{\prime}$ is established similarly. This completes the demonstration of theorem 3

\section{Comparing Approaches}

In the preceeding we have chosen to prove theorem 1 by using the result of theorem 0 , together with the scaling properties of $T_{a}^{(\nu)}, G_{a}^{(\nu)}$ and $\mathcal{E}_{t}^{(\nu)}$. In fact, it is also possible to ignore theorem 0 completely, and to prove theorem 1 as a corollary of Theorem 3. Indeed points i) and ii) of theorem 1 follow from Theorem 3 by letting $t$ tend to $+\infty$ in (45),(46) and (47), and taking expectations. In completing this agenda we show that theorem 3 also implies point iii) of theorem 1 . We shall only give the proof for $\nu>0$ and $K \geq 1$ since the other cases may be obtained in the same manner. Since it is clear that

$$
P\left(G_{\log (K)}^{(\nu)}(t)<s \mid B_{s}+\nu s=\log (x)\right)=P\left(T_{\log \left(\frac{K}{x}\right)}^{(\nu)} \geq t-s\right)
$$

we get from (46) and (61) with $K=1$ and $0<x<1$ and $t-s=u$ :

$$
\begin{aligned}
P\left(T_{\log \left(\frac{1}{x}\right)}^{(\nu)} \leq u\right) & =1-P\left(T_{\log \left(\frac{1}{x}\right)}^{(\nu)}>u\right) \\
& =1-E\left(\mathcal{E}_{u}^{(-2 \nu)} \mathbf{1}_{B_{u}^{(\nu)}>\log (x)}\right)+x^{-2 \nu} P\left(B_{u}^{(\nu)}<\log (x)\right) \\
& =1-\left(1-E\left(\mathcal{E}_{u}^{(-2 \nu)} \mathbf{1}_{B_{u}^{(\nu)}<\log (x)}\right)\right)+x^{-2 \nu} P\left(B_{u}^{(\nu)}<\log (x)\right)
\end{aligned}
$$


Hence

$$
\begin{aligned}
P\left(T_{\log \left(\frac{1}{x}\right)}^{(\nu)} \leq u\right) & =E\left(\mathcal{E}_{u}^{(-2 \nu)} \mathbf{1}_{B_{u}^{(\nu)}<\log (x)}\right)+x^{-2 \nu} P\left(B_{u}^{(\nu)}<\log (x)\right) \\
& =E\left(e^{-2 \nu B_{u}-2 \nu^{2} u} \mathbf{1}_{B_{u}+\nu u<\log (x)}\right)+x^{-2 \nu} P\left(B_{u}^{(\nu)}<\log (x)\right) \\
& =E\left(e^{2 \nu B_{u}-2 \nu^{2} u} \mathbf{1}_{-B_{u}+\nu u<\log (x)}\right)+x^{-2 \nu} P\left(B_{u}^{(\nu)}<\log (x)\right)
\end{aligned}
$$

after changing $B_{u}$ to $-B_{u}$.

We then get that

$$
P\left(T_{\log \left(\frac{1}{x}\right)}^{(\nu)} \leq u\right)=E\left(e^{2 \nu B_{u}-2 \nu^{2} u} \mathbf{1}_{B_{u}-\nu u>-\log (x)}\right)+x^{-2 \nu} P\left(B_{u}^{(\nu)}<\log (x)\right)
$$

We now replace in $(62),\left(\frac{1}{x}\right)$ by $K$, with $K \geq 1$ to obtain

$$
P\left(T_{\log (K)}^{(\nu)} \leq u\right)=E\left(\mathcal{E}_{u}^{(2 \nu)} \mathbf{1}_{B_{u}^{(-\nu)}>\log (K)}\right)+K^{2 \nu} P\left(B_{u}^{(-\nu)}>\log (K)\right)
$$

or equivalently

$$
P\left(T_{\log (K)}^{(\nu)} \leq u\right)=E\left(\mathcal{E}_{u}^{(2 \nu)} \mathbf{1}_{\mathcal{E}_{u}^{(2 \nu)}>K^{2 \nu}}\right)+K^{2 \nu} P\left(\mathcal{E}_{u}^{(2 \nu)}>K^{2 \nu}\right)
$$

We remark that we have proven (63) for $K \geq 1$ and for all $\nu$. If instead of assuming $\nu>0$ we have assumed $\nu<0$ then (63) becomes :

$$
P\left(T_{\log (K)}^{(\nu)} \leq u\right)=E\left(\mathcal{E}_{u}^{(2 \nu)} \mathbf{1}_{\mathcal{E}_{u}^{(2 \nu)}<K^{2 \nu}}\right)+K^{2 \nu} P\left(\mathcal{E}_{u}^{(2 \nu)}<K^{2 \nu}\right)
$$

\section{Conclusion}

It has been a natural question after looking at Yuri's formula (47) to give an explicit expression for

$$
P\left(G_{K}^{(\nu)}(t) \leq s \mid \mathcal{F}_{s, t}\right), \forall s<t
$$

This has been achieved in Bentata and Yor (2008) with formula (45):

$P\left(G_{K}^{(\nu)}(t) \leq s \mid \mathcal{F}_{s, t}\right)=1-\exp \left(-\frac{2}{t-s}\left[\left(B_{s}^{(\nu)}-\log (K)\right)\left(B_{t}^{(\nu)}-\log (K)\right)\right]^{+}\right)$

In fact, this formula turned out to be the key point of the whole story, since as we showed above in the proof of theorem 3 all the results found in this paper (except Theorem 2) may be derived from formula (45). 


\section{References}

[1] Akahori, J., Y. Imamura and Y. Yano (2008), "On the pricing of options written on the last exit time," Preprint.

[2] Bentata, A. and M. Yor (2008), "From Black Scholes and Dupire formulae to last passage times of local martingales," Notes from a course at the Bachelier Seminar (IHP, 02/08).

[3] Guasoni, P. (2004), "Excursions in the Martingale Hypothesis", Proceedings of the Ritsumeikan International Symposium March (2003), Stochastic Processes and Applications to Mathematical Finance, eds. Y. Akahori, S. Ogawa, S. Watanabe, World Scientific Press.

[4] Madan, D., B. Roynette and M. Yor (2008a), "Option Prices as Probabilities," Finance Research Letters, forthcoming.

[5] Madan, D., B. Roynette and M. Yor (2008b), "An alternative expression for the Black-Scholes formula in terms of first and last passage times," Preprint, 2008 no. 8 IEC Nancy.

[6] Madan, D., B. Roynette and M. Yor (2008c), "From Black-Scholes formula to local times and last passage times for certain submartingales," Preprint 2008, no. 14, IEC, Nancy.

[7] Pagès, G. (2008), "Introduction to Numerical Probability for Finance", Master Course Notes, University Paris VI.

[8] Pitman, J. (1999), "The Distribution of Local Times of a Brownian bridge," Sém. Prob. XXXIII Lecture Notes in Mathematics 1709, p. 388394. Springer Verlag, Berlin.

[9] Seshadri, V. (1988), "Exponential Models, Brownian motion and independence," Canadian Journal of Statistics, 16, 209-221.

[10] Yor, M., (1997), "Some Remarks about the joint law of Brownian motion and its supremum," Sém. Proba. XXXI, LNM 1655, 306-314. 